\title{
Building a Generation Knowledge Source using Internet-Accessible Newswire
}

\author{
Dragomir R. Radev and Kathleen R. McKeown \\ Department of Computer Science \\ Columbia University \\ New York, NY 10027 \\ \{radev,kathy\}@cs.columbia.edu
}

\begin{abstract}
In this paper, we describe a method for automatic creation of a knowledge source for text generation using information extraction over the Internet. We present a prototype system called PROFILE which uses a client-server architecture to extract noun-phrase descriptions of entities such as people, places, and organizations. The system serves two purposes: as an information extraction tool, it allows users to search for textual descriptions of entities; as a utility to generate functional descriptions (FD), it is used in a functional-unification based generation system. We present an evaluation of the approach and its applications to natural language generation and summarization.
\end{abstract}

\section{Introduction}

In our work to date on news summarization at Columbia University (McKeown and Radev, 1995; Radev, 1996), information is extracted from a series of input news articles (MUC, 1992; Grishman et al., 1992) and is analyzed by a generation component to produce a summary that shows how perception of the event has changed over time. In this summarization paradigm, problems arise when information needed for the summary is either missing from the input article(s) or not extracted by the information extraction system. In such cases, the information may be readily available in other current news stories, in past news, or in online databases. If the summarization system can find the needed information in other online sources, then it can produce an improved summary by merging information from multiple sources with information extracted from the input articles.

In the news domain, a summary needs to refer to people, places, and organizations and provide descriptions that clearly identify the entity for the reader. Such descriptions may not be present in the original text that is being summarized. For ex- ample, the American pilot Scott O'Grady, downed in Bosnia in June of 1995, was unheard of by the American public prior to the incident. If a reader tuned into news on this event days later, descriptions from the initial articles may be more useful. A summarizer that has access to different descriptions will be able to select the description that best suits both the reader and the series of articles being summarized.

In this paper, we describe a system called PROFILE that tracks prior references to a given entity by extracting descriptions for later use in summarization. In contrast with previous work on information extraction, our work has the following features:

- It builds a database of profiles for entities by storing descriptions from a collected corpus of past news.

- It operates in real time, allowing for connections with the latest breaking, online news to extract information about the most recently mentioned individuals and organizations.

- It collects and merges information from distributed sources thus allowing for a more complete record of information.

- As it parses and identifies descriptions, it builds a lexicalized, syntactic representation of the description in a form suitable for input to the FUF/SURGE language generation system (Elhadad, 1993; Robin, 1994).

The result is a system that can combine descriptions from articles appearing only a few minutes before the ones being summarized with descriptions from past news in a permanent record for future use. Its utility lies in its potential for representing entities, present in one article, with descriptions found in other articles, possibly coming from another source.

Since the system constructs a lexicalized, syntactic functional description (FD) from the extracted description, the generator can re-use the description in new contexts, merging it with other 
descriptions, into a new grammatical sentence. This would not be possible if only canned strings were used, with no information about their internal structure. Thus, in addition to collecting a knowledge source which provides identifying features of individuals, PROFILE also provides a lexicon of domain appropriate phrases that can be integrated with individual words from a generator's lexicon to flexibly produce summary wording.

We have extended the system by semantically categorizing descriptions using WordNet (Miller et al., 1990), so that a generator can more easily determine which description is relevant in different contexts.

PROFILE can also be used in a real-time fashion to monitor entities and the changes of descriptions associated with them over the course of time.

In the following sections, we first overview related work in the area of information extraction. We then turn to a discussion of the system components which build the profile database, followed by a description of how the results are used in generation. We close with our current directions, describing what parameters can influence a strategy for generating a sequence of anaphoric references to the same entity over time.

\section{Related Work}

Research related to ours falls into two main categories: extraction of information from input text and construction of knowledge sources for generation.

\subsection{Information Extraction}

Work on information extraction is quite broad and covers far more topics and problems than the information extraction problem we address. We restrict our comparison here to work on proper noun extraction, extraction of people descriptions in various information extraction systems developed for the message understanding conferences (MUC, 1992), and use of extracted information for question answering.

Techniques for proper noun extraction include the use of regular grammars to delimit and identify proper nouns (Mani et al., 1993; Paik et al., 1994), the use of extensive name lists, place names, titles and "gazetteers" in conjunction with partial grammars in order to recognize proper nouns as unknown words in close proximity to known words (Cowie et al., 1992; Aberdeen et al., 1992), statistical training to learn, for example, Spanish names, from online corpora (Ayuso et al., 1992), and the use of concept based pattern matchers that use semantic concepts as pattern categories as well as part-of-speech information (Weischedel et al., 1993; Lehnert et al., 1993). In addition, some researchers have explored the use of both lo- cal context surrounding the hypothesized proper nouns (McDonald, 1993; Coates-Stephens, 1991) and the larger discourse context (Mani et al., 1993) to improve the accuracy of proper noun extraction when large known word lists are not available. Like this research, our work also aims at extracting proper nouns without the aid of large word lists. We use a regular grammar encoding part-ofspeech categories to extract certain text patterns and we use WordNet (Miller et al., 1990) to provide semantic filtering.

Our work on extracting descriptions is quite similar to the work carried out under the DARPA message understanding program for extracting descriptions (MUC, 1992). The purpose for and the scenario in which description extraction is done is quite different, but the techniques are very similar. It is based on the paradigm of representing patterns that express the kinds of descriptions we expect; unlike previous work we do not encode semantic categories in the patterns since we want to capture all descriptions regardless of domain.

Research on a system called Murax (Kupiec, 1993) is similar to ours from a different perspective. Murax also extracts information from a text to serve directly in response to a user question. Murax uses lexico-syntactic patterns, collocational analysis, along with information retrieval statistics, to find the string of words in a text that is most likely to serve as an answer to a user's whquery. In our work, the string that is extracted may be merged, or regenerated, as part of a larger textual summary.

\subsection{Construction of Knowledge Sources for Generation}

The construction of a database of phrases for reuse in generation is quite novel. Previous work on extraction of collocations for use in generation (Smadja and McKeown, 1991) is related in that full phrases are extracted and syntactically typed so that they can be merged with individual words in a generation lexicon to produce a full sentence. However, extracted collocations were used only to determine realization of an input concept. In our work, stored phrases would be used to provide content that can identify a person or place for a reader, in addition to providing the actual phrasing.

\section{Creation of a Database of Profiles}

Figure 1 shows the overall architecture of PROFILE and the two interfaces to it (a user interface on the World-Wide Web and an interface to a natural language generation system). In this section, we describe the extraction component of PROFILE, the following section focuses on the uses of 
PROFILE for generation, and Section 7 describes the Web-based interface.

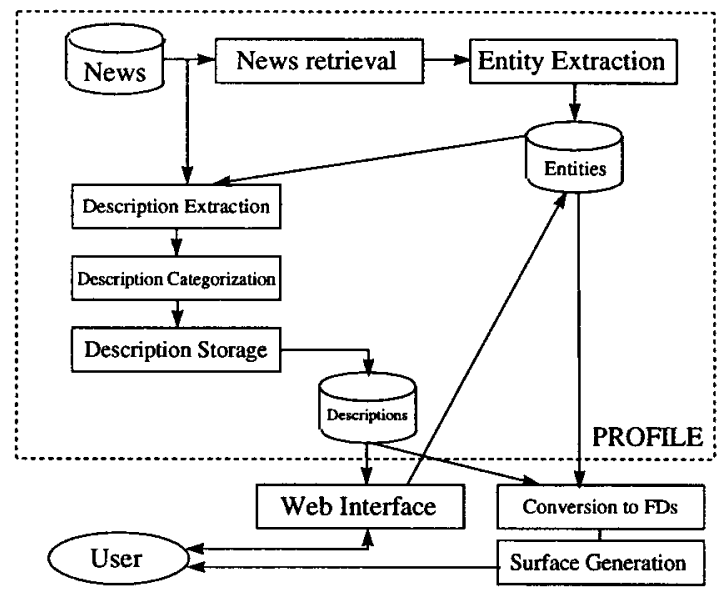

Figure 1: Overall Architecture of PROFILE.

\subsection{Extraction of entity names from old newswire}

To seed the database with an initial set of descriptions, we used a 1.4 MB corpus containing Reuters newswire from March to June of 1995 . The purpose of such an initial set of descriptions is twofold. First, it allows us to test the other components of the system. Furthermore, at the time a description is needed it limits the amount of online full text, Web search that must be done. At this stage, search is limited to the database of retrieved descriptions only, thus reducing search time as no connections will be made to external news sources at the time of the query. Only when a suitable stored description cannot be found will the system initiate search of additional text.

- Extraction of candidates for proper nouns. After tagging the corpus using the POS part-of-speech tagger (Church, 1988), we used a CREP (Duford, 1993) regular grammar to first extract all possible candidates for entities. These consist of all sequences of words that were tagged as proper nouns (NP) by POS. Our manual analysis showed that out of a total of 2150 entities recovered in this way, $1139(52.9 \%)$ are not names of entities. Among these are n-grams such as "Prime Minister" or "Egyptian President" which were tagged as NP by POS. Table 1 shows how many entities we retrieve at this stage, and of them, how many pass the semantic filtering test. The numbers in the left-hand column refer to two-word noun phrases that identify entities (e.g., "Bill Clinton"). Counts for three-word noun phrases are shown in the right-hand column. We show counts for multiple and unique occurrences of the same noun phrase.

- Weeding out of false candidates. Our system analyzed all candidates for entity names using WordNet (Miller et al., 1990) and removed from consideration those that contain words appearing in WordNet's dictionary. This resulted in a list of 421 unique entity names that we used for the automatic description extraction stage. All 421 entity names retrieved by the system are indeed proper nouns.

\subsection{Extraction of descriptions}

There are two occasions on which we extract descriptions using finite-state techniques. The first case is when the entity that we want to describe was already extracted automatically (see Subsection 3.1) and exists in PROFILE's database. The second case is when we want a description to be retrieved in real time based on a request from either a Web user or the generation system.

There exist many live sources of newswire on the Internet that can be used for this second case. Some that merit our attention are the ones that can be accessed remotely through small client programs that don't require any sophisticated protocols to access the newswire articles. Such sources include HTTP-accessible sites such as the Reuters site at www.yahoo.com and CNN Interactive at www.cnn.com, as well as others such as ClariNet which is propagated through the NNTP protocol. All these sources share a common characteristic in that they are all updated in real time and all contain information about current events. Hence, they are therefore likely to satisfy the criteria of pertinence to our task, such as the likelihood of the sudden appearance of new entities that couldn't possibly have been included a priori in the generation lexicon.

Our system generates finite-state representations of the entities that need to be described. An example of a finite-state description of the entity "Yasser Arafat" is shown in Figure 2. These full expressions are used as input to the description finding module which uses them to find candidate sentences in the corpus for finding descriptions. Since the need for a description may arise at a later time than when the entity was found and may require searching new text, the description finder must first locate these expressions in the text.

These representations are fed to CREP which extracts noun phrases on either side of the entity (either pre-modifiers or appositions) from the news corpus. The finite-state grammar for noun 


\begin{tabular}{|l|l|l|l|l|}
\hline \multirow{2}{*}{ Stage } & \multicolumn{2}{|c|}{ Two-word entities } & \multicolumn{2}{c|}{ Three-word entities } \\
\cline { 2 - 5 } & Entities & Unique Entities & Entities & Unique Entities \\
\hline POS tagging only & 9079 & 1546 & 2617 & 604 \\
After WordNet checkup & 1509 & 395 & 81 & 26 \\
\hline
\end{tabular}

Table 1: Two-word and three-word entities retrieved by the system.

SEARCH_STRING $=((\{$ NOUN_PHRASE $\}\{$ SPACE $\})+\{$ SEARCH_O $\}) \mid(\{$ SEARCH_0 $\}\{$ SPACE $\}\{$ COMMA $\}\{$ SPACE $\}$ \{NOUN_PHRASE $\})$
SEARCH_109 $=$ [Yy] asser $\{$ T_NOUN $\}\{$ SPACE $\}[$ Aa $]$ rafat $\{$ T_NOUN $\}$
SEARCH_O $=\{$ SEARCH_1 $\} \mid\{$ SEARCH_2 $\}|\ldots|\{$ SEARCH_109 $\} \mid \ldots$

Figure 2: Finite-state representation of "Yasser Arafat".

phrases that we use represents a variety of different syntactic structures for both pre-modifiers and appositions. Thus, they may range from a simple noun (e.g., "president Bill Clinton") to a much longer expression (e.g., "Gilberto Rodriguez Orejuela, the head of the Cali cocaine cartel"). Other forms of descriptions, such as relative clauses, are the focus of ongoing implementation.

Table 2 shows some of the different patterns retrieved.

\subsection{Categorization of descriptions}

We use WordNet to group extracted descriptions into categories. For all words in the description, we try to find a WordNet hypernym that can restrict the semantics of the description. Currently, we identify concepts such as "profession", "nationality", and "organization". Each of these concepts is triggered by one or more words (which we call "triggers") in the description. Table 2 shows some examples of descriptions and the concepts under which they are classified based on the WordNet hypernyms for some "trigger" words. For example, all of the following "triggers" in the list "minister", "head", "administrator", and "commissioner" can be traced up to "leader" in the WordNet hierarchy.

\subsection{Organization of descriptions in a database of profiles}

For each retrieved entity we create a new profile in a database of profiles. We keep information about the surface string that is used to describe the entity in newswire (e.g., "Addis Ababa"), the source of the description and the date that the entry has been made in the database (e.g., "reuters95_06_25"). In addition to these pieces of meta-information, all retrieved descriptions and their frequencies are also stored.

Currently, our system doesn't have the capability of matching references to the same entity that use different wordings. As a result, we keep separate profiles for each of the following: "Robert Dole", "Dole", and "Bob Dole". We use each of these strings as the key in the database of descriptions.

Figure 3 shows the profile associated with the key "John Major".

KEY: john major
SOURCE: reuters $95.03-06 .$. nws
DESCRIPTION : british prime minister
FREQUENCY: 75
DESCRIPTION : prime minister
FREQUENCY: 58
DESCRIPTION : a defiant british prime minister
FREQUENCY: 2
DESCRIPTION : his british counterpart
FREQUENCY: 1

Figure 3: Profile for John Major.

The database of profiles is updated every time a query retrieves new descriptions matching a certain key.

\section{Generation}

We have made an attempt to reuse the descriptions, retrieved by the system, in more than a trivial way. The content planner of a language generation system that needs to present an entity to the user that he has not seen previously, might want to include some background information about it. However, in case the extracted information doesn't contain a handy description, the system can use some descriptions retrieved by PROFILE.

\subsection{Transformation of descriptions into Functional Descriptions}

Since our major goal in extracting descriptions from on-line corpora was to use them in generation, we have written a utility which converts finite-state descriptions retrieved by PROFILE into functional descriptions (FD) (Elhadad, 1991) that we can use directly in generation. A description retrieved by the system from the article in 4 is shown in Figure 5. The corresponding FD is shown in Figure 6.

We have implemented a TCP/IP interface to Surge. The FD generation component uses this interface to send a new FD to the surface realization component of Surge which generates an English surface form corresponding to it. 


\begin{tabular}{|l|l|l|}
\hline Example & Trigger Term & Semantic Category \\
\hline Addis Ababa, the Ethiopian capital & capital & location \\
South Africa's main black opposition leader, Mangosuthu Buthelezi & leader & occupation \\
Boerge Ousland, 33 & 33 & age \\
maverick French ex-soccer boss Bernard Tapie & boss & occupation \\
Italy's former prime minister, Silvio Berlusconi & minister & occupation \\
Sinn Fein, the political arm of the Irish Republican Army & arm & organization \\
\hline
\end{tabular}

Table 2: Examples of retrieved descriptions.

MILAN - A judge ordered Italy's former prime minister Silvio Berlusconi to stand trial in January on corruption charges in a ruling that could destroy the media magnate's hope of returning to high office.

Figure 4: Source article.

Italy@NPNP 'sQ\$ former $0 J J$ primeQJJ

minister@NN Silvio@NPNP Berlusconi@NPNP

Figure 5: Retrieved description for Silvio Berlusconi.

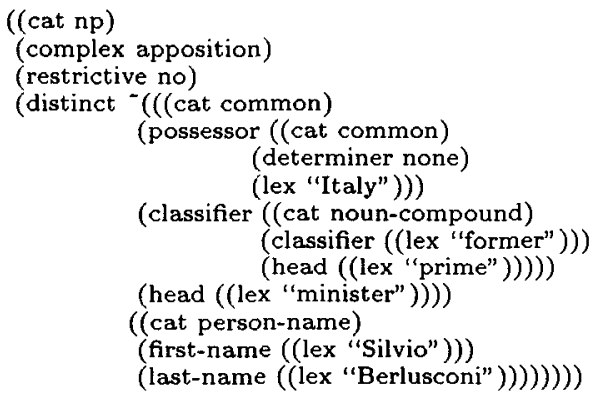

Figure 6: Generated FD for Silvio Berlusconi.

\subsection{Lexicon creation}

We have identified several major advantages of using FDs produced by the system in generation compared to using canned phrases.

- Grammaticality. The deeper representation allows for grammatical transformations, such as aggregation: e.g., "president Yeltsin" + "president Clinton" can be generated as "presidents Yeltsin and Clinton".

- Unification with existing ontologies. E.g., if an ontology contains information about the word "president" as being a realization of the concept "head of state", then under certain conditions, the description can be replaced by one referring to "head of state".

- Generation of referring expressions. In the previous example, if "president Bill Clinton" is used in a sentence, then "head of state" can be used as a referring expression in a subsequent sentence.

- Enhancement of descriptions. If we have retrieved "prime minister" as a description for Silvio Berlusconi, and later we obtain knowledge that someone else has become Italy's primer minister, then we can generate "former prime minister" using a transformation of the old FD.

- Lexical choice. When different descriptions are automatically marked for semantics, PROFILE can prefer to generate one over another based on semantic features. This is useful if a summary discusses events related to one description associated with the entity more than the others.

- Merging lexicons. The lexicon generated automatically by the system can be merged with a domain lexicon generated manually.

These advantages look very promising and we will be exploring them in detail in our work on summarization in the near future.

\section{Coverage and Limitations}

In this section we provide an analysis of the capabilities and current limitations of PROFILE.

\subsection{Coverage}

At the current stage of implementation, PROFILE has the following coverage.

- Syntactic coverage. Currently, the system includes an extensive finite-state grammar that can handle various pre-modifiers and appositions. The grammar matches arbitrary noun phrases in each of these two cases to the extent that the POS part-of-speech tagger provides a correct tagging.

- Precision. In Subsection 3.1 we showed the precision of the extraction of entity names. Similarly, we have computed the precision of retrieved 611 descriptions using randomly selected entities from the list retrieved in Subsection 3.1. Of the 611 descriptions, 551 $(90.2 \%)$ were correct. The others included a roughly equal number of cases of incorrect NP attachment and incorrect part-of-speech 
assignment. For our task (symbolic text generation), precision is more important than recall; it is critical that the extracted descriptions are correct in order to be converted to FD and generated.

- Length of descriptions. The longest description retrieved by the system was 9 lexical items long: "Maurizio Gucci, the former head of Italy's Gucci fashion dynasty". The shortest descriptions are 1 lexical item in length e.g. "President Bill Clinton".

- Protocol coverage. We have implemented retrieval facilities to extract descriptions using the NNTP (Usenet News) and HTTP (World-Wide Web) protocols.

\subsection{Limitations}

Our system currently doesn't handle entity crossreferencing. It will not realize that "Clinton" and "Bill Clinton" refer to the same person. Nor will it link a person's profile with the profile of the organization of which he is a member.

At this stage, the system generates functional descriptions (FD), but they are not being used in a summarization system yet.

\section{Current Directions}

One of the more important current goals is to increase coverage of the system by providing interfaces to a large number of on-line sources of news. We would ideally want to build a comprehensive and shareable database of profiles that can be queried over the World-Wide Web.

We need to refine the algorithm to handle cases that are currently problematic. For example, polysemy is not properly handled. For instance, we would not label properly noun phrases such as "Rice University", as it contains the word "rice" which can be categorized as a food.

Another long-term goal of our research is the generation of evolving summaries that continuously update the user on a given topic of interest. In that case, the system will have a model containing all prior interaction with the user. To avoid repetitiveness, such a system will have to resort to using different descriptions (as well as referring expressions) to address a specific entity ${ }^{1}$. We will be investigating an algorithm that will select a proper ordering of multiple descriptions referring to the same person.

After we collect a series of descriptions for each possible entity, we need to decide how to select

\footnotetext{
${ }^{1}$ Our corpus analysis supports this proposition a large number of threads of summaries on the same topic from the Reuters and UPI newswire used up to 10 different referring expressions (mostly of the type of descriptions discussed in this paper) to refer to the same entity.
}

among all of them. There are two scenarios. In the first one, we have to pick one single description from the database which best fits the summary that we are generating. In the second scenario, the evolving summary, we have to generate a sequence of descriptions, which might possibly view the entity from different perspectives. We are investigating algorithms that will decide the order of generation of the different descriptions. Among the factors that will influence the selection and ordering of descriptions, we can note the user's interests, his knowledge of the entity, the focus of the summary (e.g., "democratic presidential candidate" for Bill Clinton, vs. "U.S. president"). We can also select one description over another based on how recent they have been included in the database, whether or not one of them has been used in a summary already, whether the summary is an update to an earlier summary, and whether another description from the same category has been used already. We have yet to decide under what circumstances a description needs to be generated at all.

We are interested in implementing existing algorithms or designing our own that will match different instances of the same entity appearing in different syntactic forms - e.g., to establish that "PLO" is an alias for the "Palestine Liberation Organization". We will investigate using cooccurrence information to match acronyms to full organization names and alternative spellings of the same name with each other.

An important application that we are considering is applying the technology to text available using other protocols - such as SMTP (for electronic mail) and retrieve descriptions for entities mentioned in such messages.

We will also look into connecting the current interface with news available to the Internet with an existing search engine such as Lycos (www.lycos.com) or AltaVista (www.altavista.digital.com). We can then use the existing indices of all Web documents mentioning a given entity as a news corpus on which to perform the extraction of descriptions.

Finally, we will investigate the creation of KQML (Finin et al., 1994) interfaces to the different components of PROFILE which will be linked to other information access modules at Columbia University.

\section{Contributions}

We have described a system that allows users to retrieve descriptions of entities using a Web-based search engine. Figure 7 shows the Web interface to PROFILE. Users can select an entity (such as "John Major"), specify what semantic classes of descriptions they want to retrieve (e.g., age, posi- 
tion, nationality) as well as the maximal number of queries that they want. They can also specify which sources of news should be searched. Currently, the system has an interface to Reuters at www.yahoo.com, The CNN Web site, and to all local news delivered via NNTP to our local news domain.

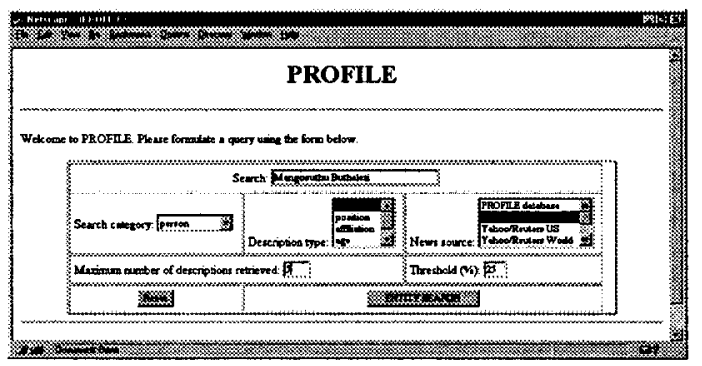

Figure 7: Web-based interface to PROFILE.

The Web-based interface is accessible publicly (currently within Columbia University only). All queries are cached and the descriptions retrieved can be reused in a subsequent query. We believe that such an approach to information extraction can be classified as a collaborative database.

The FD generation component produces syntactically correct functional descriptions that can be used to generate English-language descriptions using FUF and Surge, and can also be used in a general-purpose summarization system in the domain of current news.

All components of the system assume no prior domain knowledge and are therefore portable to many domains - such as sports, entertainment, and business.

\section{Acknowledgments}

This work was partially supported by NSF grant GER-90-2406 and by a grant from Columbia University's Strategic Initiative Fund sponsored by the Provost's Office.

\section{References}

John Aberdeen, John Burger, Dennis Connolly, Susan Roberts, and Marc Vilain. 1992. Mitrebedford: Description of the alembic system as used for muc-4. In Proceedings of the Fourth
Message Understanding Conference (MUC-4), pages 215-222, McLean, Virginia, June.

Damaris Ayuso, Sean Boisen, Heidi Fox, Herb Gish, Robert Ingria, and Ralph Weischedel. 1992. Bbn: Description of the plum system as used for muc-4. In Proceedings of the Fourth Message Understanding Conference (MUC-4), pages 169-176, McLean, Virginia, June.

Kenneth W. Church. 1988. A stochastic parts program and noun phrase parser for unrestricted text. In Proceedings of the Second Conference on Applied Natural Language Processing (ANLP-88), pages 136-143, Austin, Texas, February. Association for Computational Linguistics.

Sam Coates-Stephens. 1991. Automatic lexical acquisition using within-text descriptions of proper nouns. In Proceedings of the Seventh Annual Conference of the UW Centre for the New OED and Text Research, pages 154-169.

Jim Cowie, Louise Guthrie, Yorick Wilks, James Pustejovsky, and Scott Waterman. 1992. $\mathrm{Crl} / \mathrm{nmsu}$ and brandeis: Description of the mucbruce system as used for muc-4. In Proceedings of the Fourth Message Understanding Conference (MUC-4), pages 223-232, McLean, Virginia, June.

Darrin Duford. 1993. Crep: a regular expressionmatching textual corpus tool. Technical Report CUCS-005-93, Columbia University.

Michael Elhadad. 1991. Fuf: The universal unifier - user manual, version 5.0. Technical Report CUCS-038-91, Columbia University.

Michael Elhadad. 1993. Using argumentation to control lexical choice: a unification-based implementation. Ph.D. thesis, Computer Science Department, Columbia University.

Tim Finin, Rich Fritzson, Don McKay, and Robin McEntire. 1994. KQML - a language and protocol for knowledge and information exchange. Technical Report CS-94-02, Computer Science Department, University of Maryland and Valley Forge Engineering Center, Unisys Corporation.

R. Grishman, C. Macleod, and J. Sterling. 1992. New york university: Description of the proteus system as used for muc-4. In Proceedings of the Fourth Message Understanding Conference, June.

Julian M. Kupiec. 1993. Murax: A robust linguistic approach for question answering using an on-line encyclopedia. In Proceedings, 16th Annual International ACM SIGIR Conference on Research and Development in Information Retrieval. 
W. Lehnert, J. McCarthy, S. Soderlan, E. Riloff, C. Cardie, J. Peterson, and F. Feng. 1993. Umass/hughes: Description of the circus system used for muc-5. In Proceedings of the Fifth Message Understanding Conference (MUC-5), pages 277-291, Baltimore, Md., August.

Inderjeet Mani, Richard T. Macmillan, Susann Luperfoy, Elaine Lusher, and Sharon Laskowski. 1993. Identifying unknown proper names in newswire text. In Proceedings of the Workshop on Acquisition of Lexical Knowledge from Text, pages 44-54, Columbus, Ohio, June. Special Interest Group on the Lexicon of the Association for Computational Linguistics.

David D. McDonald. 1993. Internal and external evidence in the identification and semantic cateogrization of proper names. In Proceedings of the Workshop on Acquisition of Lexical Knowledge from Text, pages 32-43, Columbus, Ohio, June. Special Interest Group on the Lexicon of the Association for Computational Linguistics.

Kathleen R. McKeown and Dragomir R. Radev. 1995. Generating summaries of multiple news articles. In Proceedings, 18th Annual International ACM SIGIR Conference on Research and Development in Information Retrieval, pages 74-82, Seattle, Washington, July.

George A. Miller, Richard Beckwith, Christiane Fellbaum, Derek Gross, and Katherine J. Miller. 1990. Introduction to WordNet: An on-line lexical database. International Journal of Lexicography (special issue), 3(4):235-312.

Message Understanding Conference MUC. 1992. Proceedings of the Fourth Message Understanding Conference (MUC-4). DARPA Software and Intelligent Systems Technology Office.

Woojin Paik, Elizabeth D. Liddy, Edmund Yu, and Mary McKenna. 1994. Interpretation of proper nouns for information retrieval. In Proceedings of the Human Language Technology Workshop, pages 309-313, Plainsboro, New Jersey, March. ARPA Software and Intelligent Systems Technology Office, Morgan Kaufmann.

Dragomir R. Radev. 1996. An architecture for distributed natural language summarization. In Proceedings of the 8th International Workshop on Natural Language Generation: Demonstrations and Posters, pages 45-48, Herstmonceaux, England, June.

Jacques Robin. 1994. Revision-Based Generation of Natural Language Summaries Providing Historical Background. Ph.D. thesis, Computer Science Department, Columbia University.
Frank Smadja and Kathleen R. McKeown. 1991. Using collocations for language generation. Computational Intelligence, 7(4), December.

Ralph Weischedel, Damaris Ayuso, Sean Boisen, Heidi Fox, Robert Ingria, Tomoyoshi Matsukawa, Constantine Papageorgiou, Dawn MacLaughlin, Masaichiro Kitagawa, Tsutomu Sakai, June Abe, Hiroto Hosihi, Yoichi Miyamoto, and Scott Miller. 1993. Bbn: Description of the plum system as used for muc5. In Proceedings of the Fifth Message Understanding Conference (MUC-5), pages 93-108, Baltimore, Md., August. 